Original Article

\title{
Evaluation of Polymerization shrinkage of 2 types of posterior composite resins
}

\author{
Lakshmi Nidhi Rao ${ }^{1}$, Mithra N. Hegde ${ }^{2}$, Aditya Shetty ${ }^{3}$
}

${ }^{1}$ Lecturer, ${ }^{2}$ Vice-Principal,Senior Professor and Head of the Department, ${ }^{3}$ Add. Professor, Department of Conservative Dentistry and Endodontics, A.B. Shetty M emorial Institute of Dental Sciences, Deralakatte, M angalore.

*Corresponding Author : Lakshmi Nidhi Rao, Dimpi Cottage, 2 ${ }^{\text {nd }}$ Bridge,Jeppu, Mangalore- 575 002, E-mail : dimpirao@ rediffmail.com

Received

: 02.02.2017

Review Completed : 13.02.2017

Accepted

: 18.02 .2017

Keywords : Composite, Polymerization shrinkage, Pycnometer

\begin{tabular}{|c|}
\hline Access this article online \\
\hline Quick Response Code \\
\hline
\end{tabular}

\begin{abstract}
Composite resins represent a class of material widely used in restorative dentistry, not only for anterior aesthetics but also as the first choice to restore posterior teeth. However the key limitation in the use of composite resins as a restorative material is related to shrinkage during polymerization which leads to poor marginal seal, marginal staining, restoration displacement, tooth fracture and recurring caries [1].

Polymerization shrinkage may affect negatively the clinical outcome of the restoration. Hence the present study evaluates the Polymerization shrinkage of 2 different posterior composites; FiltekZ350(3M) and everX Posterior(GC), using a pycnometer.

Independent Sample T-Test was used to determine statistically significant difference in volumetric shrinkage among the tested composite resins. everX Posterior showed comparatively less shrinkage than Filtek Z350; which can be attributed to the presence of silanated e-glassfibres.
\end{abstract}

\section{Introduction}

Composite resins have traditionally been the preferred material for aesthetic restorations. In the last few decades, adhesive dentistry has evolved tremendously owing to the development and incorporation of new initiation systems, new monomers and filler technologies which help to improve the physical properties of the materials [1].

Among the key factors related to the clinical outcome of composite resin restorations; tooth interfacial seal, restorative material quality and absence of micro leakage play a very critical role $[2,3]$. This is largely determined by the quality of bonding to tooth structures and the amount of polymerization shrinkage of composite resin [4]. There are other critical factors which are well known to contribute to the quality of marginal sealing, key among them being filling technique, configuration factor, bonding system and modulation of light curing [5].

Polymerization of the material is determined by the degree of conversion of monomers into polymers indicating the number of methacrylate groups that have reacted with each other during conversion process. The shrinkage suffered by the composite during curing ranges from 1.35\% to $7.1 \%$ [6]. The longevity and function of the restoration depends on dimensional stability of composites. Dental composite resins primarily consist of a dimethacrylate resin filled with organic or inorganic filler particles and upon cure the dimethacrylate matrix undergoes a volumetric shrinkage [7]. As a result of this shrinkage, contraction related stresses are generated within the material, which in turn cause mechanical failure at the composite tooth interface $[8,9]$ or the formation of marginal gaps permitting the ingress of bacteria into the cavity. Thus low polymerization shrinkage is generally seen as a key performance indicator for this material genre [10].

To bring about significant advances to improve the properties of polymer matrix improvements in dental composite properties have been brought about. Composite materials in general can be either fibre or 
particle reinforced $[11,12]$. Short fibre reinforced composite (everX Posterior) was introduced as a restorative composite resin [13]. It consists of a combination of a resin matrix, silanated e-glass fibers and inorganic particulate fillers $[14,15]$.

A universal demand for restorative material for all the types of direct restorations including posterior teeth, resulted in development of another category of resin composite called nano filled composites; Filtek Z350 being one of them. Filtek Z350 exhibits sufficient compressive strength and wear resistance to justify their use in high stress-bearing areas, such as the occlusal surfaces of posterior teeth [16, 17].

The present study evaluates the volumetric polymerization shrinkage of these two posterior composite resins using a Pycnometer.

\section{Materials and Methods}

Twenty samples of Composite resin were prepared; Group 1 consisted of 10 samples using Filtek Z350 XT Universal (3M) and Group 2 consisted of 10 samples using everX Posterior (GC). The tests were carried out in a standard laboratory under controlled room temperature $(23 \pm 3 \circ \mathrm{C})$ and relative humidity. The density of the material was determined using a Pycnometer.

To perform the test, $1 \mathrm{~g}$ of composite resin, as recommended by ASTM: D792 specification[18], was placed in a Teflon split mould and pressed manually with two flat glass plates resulting in a thin disc ( $\pm 2.0 \mathrm{~mm}$ thick). The Pycnometer was filled with distilled water and placed in a water bath until temperature equilibrium with the water bath is obtained. The weight of the Pycnometer filled with water is determined. After cleaning and drying the Pycnometer, $1 \mathrm{~g}$ of Composite material is added and the combined weight of the specimen and the Pycnometer is determined. The Pycnometer is now filled with water. Finally the weight of the Pycnometer filled with water and specimen is recorded. According to Puckett; Smith [19], no alteration in the weight of resin occurs when they are submerged in water for up to 2 minutes before polymerization.

Using the values of the weight obtained, specific gravity is calculated using the formula;

Specific gravity $=\frac{a}{(b+a)-m}$

Where,

$a=$ weight of the specimen

$b=$ weight of the Pycnometer filled with water

$m=$ weight of the Pycnometer containing the specimen and water.

The specimen is removed from water, dried with absorbent paper and then polymerized with a light-curing unit (Elipar LED light curing unit) for 20 seconds. After polymerization the same procedure as mentioned is repeated and Specific gravity of the polymerized samples were obtained.

The volume of the specimens (pre and postpolymerization) is calculated as follows;

Specific gravity = Density of the Specimen / Density of water;

Where Density of water is a constant at $1 \mathrm{gm} / \mathrm{cm}^{3}$

Volume of the specimen is calculated as;

Volume $=$ Mass $/$ Density of the Specimen .

After obtaining the pre and post-polymerization volumes of the specimens, the percentage of volumetric contraction was calculated as follows:

Percentage shrinkage $=(\mathrm{V} 1-\mathrm{V} 2 / \mathrm{V} 2) \times 100$

Where,

V1 =Volume of unpolymerized resin;

V2 =Volume of polymerized resin

Independent Sample T-Test was used to determine statistically significant difference in volumetric shrinkage among the tested composite resins.

\section{Results}

Filtek Z350 presented higher polymerization shrinkage of $1.87 \%$ compared to everX Posterior which showed shrinkage of $0.94 \%$ ( $p$-value $\varangle 0.01$ ). 


\begin{tabular}{|l|c|c|c|c|c|c|c|}
\hline & N & Mean & SD & Mean difference (95\% Cl) & t & df & p-value \\
\hline Group 1 (Filtek Z350) & 10 & 1.87 & 0.41 & $0.94(0.66-1.21)$ & 7.078 & 18 & \multirow{2}{*}{$4.01^{*}$} \\
\cline { 1 - 4 } Group 2 (everX Posterior) & 10 & 0.94 & 0.07 & & & & \\
\hline
\end{tabular}

Table 1: Volumetric Polymerization Shrinkage

$* p \varangle 0.05$ statistically significant, $p>0.05$ non significant

\section{Discussion}

Despite continued advancement in adhesive dentistry, polymerization shrinkage of resin composites still remains a major challenge [20]. The polymerization shrinkage produced in a given composite resin is known to be related to the opacity, shade and composition of the composite resin, exposure times of curing light used, incompatibility between a photo-initiator system and spectral output of the curing light, composite layer thickness and cavity preparation geometry $[21,22]$.

Polymerization shrinkage can gradually lead to marginal microleakage caused due to the detachment of the composite material from cavity margins. This allows the passage of micro-organisms, molecules, fluids between the cavo surface margin and the composite resin [23]. Microleakage of posterior composite restorations is a matter of concern to the clinician, as it leads to secondary caries, pulp pathology, staining at the margins of restorations and hypersensitivity [24].

In the present study, polymerization shrinkage of two dentin replacement composites were evaluated, namely; a short fibre reinforced composite - everX Posterior and a nanocomposite - Filtek Z350 XT Universal restorative material using a Pycnometer.

The results obtained in the present study showed polymerization shrinkage to be higher in Group I:Filtek Z350 as compared to Group II:everX Posterior. This can be attributed to the reinforcement of short fibres in everX Posterior. The fibre length plays an important role in a restorative composite resin. According to Cheng $\mathrm{TH}$ et al [25], the critical fibre length with Bis-GMA polymer matrix varies between 0.5 and $1.6 \mathrm{~mm}$. In order for the fibre to act as effective reinforcement for polymers, stress transfer from the polymer matrix to the fibres is essential. This is achieved by having a fibre length equal to or greater than the critical fibre length [26, 27]. everX Posterior has a fibre length of $1 \mathrm{~mm}$ to $2 \mathrm{~mm}$ thus exceeding the critical fibre length. This contributes to substantial improvements in its physical properties. During placement into the cavity, the fibres orientate into a horizontal plane within the cavity. Due to strong adhesion between resin and silanated fibres in everX posterior the direction of the fibres minimizes polymerization shrinkage.

The polymerization shrinkage of a composite resin is inversely proportional to the monomer composition. The monomeric composition of filtek Z350 is BisGMA, BisEM A and UDMA, each of them being high molecular weight monomers with high viscosity. Due to its high viscosity it requires dilution with low viscosity dimethacrylates such as TEGDM A, however as a result of the incorporation of diluent monomers during polymerization the resin system shrinks mainly because the intermolecular distance of the monomer molecules in the network shortens when the double bonds are polymerized to covalent main chain bonds[20].

In the present study, the polymerization shrinkage was measured using the pycnometer method as it is a simple method for the measurement of polymerization shrinkage of dental composite. This method involves measuring density of the material by measuring its mass and volume. Using mathematical formulas mentioned the change in density and corresponding polymerization shrinkage was calculated using the values of specific gravity obtained. This method is based on the hypotheses of Archimedes's principle which states that "a body immersed in a fluid is subject to an upward force due to which its mass decreases proportionally to the mass of the fluid it displaces". The change in density and corresponding polymerization shrinkage was calculated.

Our study has demonstrated that short glass fiber 
reinforced composite resin everX Posterior showed lower polymerization shrinkage compared to Filtek Z350. This can be attributed to the strong adhesion between resin and silanated e-glass fibers. Additionally the direction of the fibres minimizes shrinkage in the horizontal plane after placement. This could derive better performance and durability in posterior restorations [28].

\section{Conclusion}

Within the limitations of this study, everX Posterior showed

\section{References}

1. Kevin Donly et al. Relationship among visible light source, composite resin polymerization shrinkage and hygroscopic expansion. Quintessence International 1990; Volume 21, Number 11: 883-86.

2. Alani AH, Toh CG. Detection of microleakage around dental materials: a review. Oper Dent. 1997;22:173-85.

3. Tulunoglu Ö, Tulunoglu I, Ulusu T, Genç Y. Penetration of radiocalcium at the margins of resin and glass ionomer dentine agents in primary $e$ permanent teeth. J Dent. 2000;7:481-6.

4. Winkler MM. Experimental validation of a finite element model of light-activated polymerization shrinkage. J Biomed Mater Res.2000;53:554-9.

5. Fares NH, Coutinho KQ, Couto MG, Couto-Júnior M, Nagem-Filho H. Tensões de Contração das Resinas Compostas Geradas durante a Polimerização. Rev Port Estomatol Cir M axilofac. 2004:45:177-84.

6. Polymerization Shrinkage of Composite Resins: A Review, Dr. Akshay Langalia, Dr Aastha Buch, Malhar Khamar, Dr. Parth Patel, Quest Journals, Journal of M edical and Dental Science Research, Volume 2 Issue 10 (2015) pp: 23-27.

7. Cook WD, Beech DR, Tyas MJ. Structure and properties of methacrylate-based dental restorative materials, Biomaterials 1985,6:362-368

8. Bausch JR, de Lange K, C.L. Davidson A, Peters AJ. Clinical significance of polymerization shrinkage of composite resins. J Prosth Dent 1982; 48;59-67.

9. Bandyopadhyay S, Study of volumetric setting shrinkage of some dental materials.J BioM ed M ater Res 1982; 16;135-144.

10.W.D.Cook, M.Forrest, A.A.Goodwin A simple method for measurement of polymerization shrinkage in dental composites, Elsevier, Dental M aterials 15(1999) 447-449.

11. Xu HHK, Quinn JB, Smith DT, Giuseppetti AA, Eichmiller FC. Effect of different whiskers on the reinforcement of dental resin composites. Dental M aterials 2003;19:359-67.

12. Garoushi S, Vallittu PK, Lassila LVJ. Short glass fiber reinforced restorative composite resin with semi-interpenetrating polymer network matrix. Dental M aterials 2007;23:1356-62.

13. Garoushi S, Tanner J, Vallittu PK, Lassila LV]. Preliminary clinical evaluation of short fiber-reinforced composite resin in posterior teeth: 12-months report. The Open Dentistry Journal 2012;6:41-5.

14. Lastumäki TM, Lassila LV, Vallittu PK. The semi-interpenetrating polymer network matrix of fiber-reinforced composite and its effect on the surface adhesive properties. Journal of Materials Science: M aterialsin Medicine 2003:14:803-9. comparatively less shrinkage than Filtek Z350 which can be attributed to the presence of silanated e-glass fibres. Since polymerization shrinkage compromises the success and longevity of the restoration, it becomes imperative for the clinician to choose composite resin with lower polymerization shrinkage rates for the success of the restoration.

15. Vallittu PK. Interpenetrating polymer networks (IPNs) in dental polymers and composites. In: Matinlinna JP, Mittal KL, editors. Adhesion aspects in dentistry. Leiden, The Netherlands: VSP; 2009. p. 6-74.

16. Cetin AR, \& Unlu N (2009) One-year clinical evaluation of direct nanofilled and indirect composite restorations in posterior teeth Dental M aterials Journal 28(5) 620-626.

17. Dresch W, Volpato S, Gomes JC, Ribeiro NR, Reis A, \& Loguercio AD (2006) Clinical evaluation of a nanofilled composite in posterior teeth: 12-month results Operative Dentistry 31(4) 409-417.

18. American for testing and materials. Standard test methods for density and specific gravity (relative density) of plastics by displacement (ASTM) D 792-98.

19. Puckett AD, Smith R. M ethod to measure the polymerization shrinkage of light-cured composites. J Prosthet Dent. 1992;68;56-8.

20. Xavier, Janaína Cavalcanti, Monteiro, Gabriela Queiroz de Melo, \& Montes, Marcos Antonio Japiassú Resende. (2010). Polymerization Shrinkage and Flexural Modulus of Flowable Dental Composites. M aterials Research, 13(3), 380-384

21. Rueggeberg FA, Caughman WF, Curtis JW Jr. Effect of light intensity and exposure duration on cure of resin composite. Oper Dent. 1994;19: 26-32.

22. Alster D, Venhoven BA, Feilzer AJ, et al. Infuence of compliance of the substrate materials on polymerization contraction stress in thin resin composite layers. Biomaterials. 1997;18(4):337-341.

23. Kidd EA, Beighton D (1996) Prediction of secondary caries around tooth-colored restorations: A clinical and microbiological study Journal of Dental Research 75(12) 1942-1946.

24. Going RE (1972) Microleakage around dental restorations: A summarizing review Journal of the American Dental Association 84(6) 1349-57.

25. Cheng TH, Jones FR, Wang D. Effect of fiber conditioning on the interfacial shear strength of glass-fiber composite. Compos Sci Technol. 1993;48:89-96.

26. Petersen RC. Discontinuous fiber-reinforced composites above critical length.] Dent Res. 2005;84:365-70. [PM Cfree article] [PubM ed]

27. Vallittu PK, Lassila VP, Lappalainen R. Transverse strength and fatigue of denture acrylic-glass fiber composite. Dent M ater. 1994;10:116-21. [PubMed]

28. Sufyan Garoushi, Eija Säilynoja, Pekka K. Vallittu, Lippo V.J. Lassila, Physical properties and depth of cure of a new short fiber reinforced composite, Elsevier, 29(2013), 835-841. 